\title{
SECONDARY BREAST TUBERCULOSIS: A CASE REPORT
}

\author{
Ankur Sharma1, Goonj Johri² \\ ${ }^{1}$ Assistant Professor, Department of General Surgery, IIMS\&R, Integral University, Lucknow. \\ ${ }^{2}$ Assistant Professor, Department of General Surgery, Era's Lucknow Medical College, Lucknow.
}

\begin{abstract}
Tuberculosis of the breast is a rare disease which is not often seen in day to day clinical practice. Breast Tuberculosis can be primary or secondary to some focus in body. A case of secondary tuberculosis of right breast in a 16-year-old female from India is being reported. She presented with multiple painless discharging sinuses in the right breast. Tubercular osteomyelitis of $5^{\text {th }}$ and $6^{\text {th }}$ ribs was the source of secondary breast involvement. No evidence of pulmonary tuberculosis was present. Excision biopsy of the sinus tract along with curettage of the ribs was done and patient recovered fully with 9 months of ATT.
\end{abstract}

KEYWORDS: Breast, Secondary Tuberculosis, Rib Osteomyelitis.

HOW TO CITE THIS ARTICLE: Ankur Sharma, Goonj Johri. "Secondary Breast Tuberculosis: A Case Report". Journal of Evolution of Medical and Dental Sciences 2015; Vol. 4, Issue 92, November 16; Page: 15807-15808, D0I: 10.14260/jemds/2015/2286.

INTRODUCTION: Tuberculosis has always remained a major public health concern both in developing and developed countries. Immunocompromised states like HIV have seen resurgence in cases of Tuberculosis, both pulmonary and Extra-pulmonary. India contributes to around $26 \%$ of global TB burden. While pulmonary tuberculosis is the most common presentation, extrapulmonary tuberculosis (EPTB) is also an important clinical problem, often difficult to prove due to paucibacillary nature. ${ }^{1}$ The incidence of tuberculosis of breast is less than $1 \%$, probably due to inherent resistance of breast tissue to tubercular bacteria but is also under reported in literature. ${ }^{2}$

CASE REPORT: A 16years old girl from a low socio-economic status presented with complaint of multiple discharging sinuses from right breast of 1 year duration. There was a history of nodule in right breast preceding the sinus, which spontaneously burst open and formed a sinus discharging pus. Subsequently she developed one more sinus on the same side. Patient gave history of evening rise of temperature, decreased appetite and weight loss of around $10 \mathrm{kgs}$ in one year. There was no history of pain or nipple discharge or contact with tuberculosis. She was treated elsewhere with a course of antibiotics and ATT. She took treatment for 1 month and then discontinued.

On Clinical examination, 2 sinus openings (Fig 1) were noted on the outer margin of right breast towards the inferior and lateral side and marked induration around them. No underlying lump was palpable. Two firm tracts were probed and found communicating with ribs. There was no axillary lymphadenopathy. ESR was raised. Mantoux test was indeterminate. Serostatus for HIV was negative. Chest $\mathrm{X}$ ray showed osteomyelitic changes in $5^{\text {th }}$ and $6^{\text {th }}$ ribs with resorption of bone on right side (Fig 2). A ZN Stain done from the discharge was negative for AFB.

Financial or Other, Competing Interest: None.

Submission 24-10-2015, Peer Review 26-10-2015,

Acceptance 04-11-2015, Published 16-11-2015.

Corresponding Author:

Dr. Ankur Sharma,

Department of General Surgery,

IIMS\&R Integral University, Dasauli,

Lucknow-226026.

E-mail: ankurvashishth@gmail.com

DOI:10.14260/jemds/2015/2286
Excision of both sinus fibrous (Fig 3) tracts along with curettage of ribs was done and HPE examination revealed chronic granulomatous infection. Although Histopathology was not specific for tuberculosis, but in view of widespread nature of TB in India, and past history of incomplete ATT, she was again started on standard 4 drug ATT to which patient showed response and within 6 weeks of ATT, the lesions had started to heal and by the end of 3 months, both lesions had healed completely. Meanwhile, she was counselled aggressively regarding completion of treatment and was kept under strict follow up. Patient successfully completed 9 months Category II DOTS with no recurrence of disease in last 1 year.

DISCUSSION: Sir Astley Cooper in 1829 first described breast TB as a scrofulous swelling at the bosom of young women suffering from enlargement of cervical glands. ${ }^{3}$ Lack of awareness and manifestations simulating benign and malignant lesions of breast are contributory to overlooking and misdiagnosis of breast tuberculosis. Also it may coexist with carcinoma. High incidence of breast tuberculosis is presumed in India despite only few hundred cases of breast tuberculosis reported. 4 No well-defined clinical features suggestive of breast tuberculosis are defined. Essentially, tuberculous infection is rarely confined only to the breast, and secondary tuberculosis is sequela of tuberculosis in other organs of the body.

Risk factors are multiparity, lactation, trauma, past history of suppurative mastitis, and AIDS. Various routes of infection described, including haematogenous, lymphatic, direct inoculation, and/or ductal infection. Occasionally, direct extension from contiguous structures such as infected rib, costochondral cartilage, sternum, shoulder joint, through the chest wall from a tuberculous pleurisy, or via abrasions in the skin can pass infection into breast. ${ }^{5}$

Tuberculosis involving ribs and presenting with breast mass is a very rare entity, and only a few cases have been to date reported in the literature. A direct fistulous tract with the pleura or a destroyed rib fragment in the abscess can be seen due to disease. ${ }^{6}$ Symptoms in breast tuberculosis are usually seen for less than one year. Breast tuberculosis can present as a lump, ulcer, and breast abscess with or without discharging sinuses. Lump is commonly seen in the central or upper outer quadrant of the breast, an extension of tuberculosis from axillary nodes to the breast. 
Formation of fistulas and sinus tracts is usually seen in advanced disease or after needle puncture. ${ }^{7} \mathrm{X}$-ray chest is done to detect any evidence of active or stigmata of healed lesion in the lungs. Diagnosis is based on identification of typical histological features or the tubercle bacilli under microscopy or culture. 8 Microscopy shows fibrous and lymphoid tissue infiltrated by large epithelioid granulomas with central acellular necrosis and many giant cells.The culture used to be positive only in $25 \%-30 \%$ cases. In mammography, three different patterns are recognized-the nodular pattern, the disseminated pattern and the sclerosing pattern. It is difficult to differentiate between tuberculous lesion and carcinoma on mammogram.

Ultrasonography reveals heterogeneous, hypoechoic, fluid containing masses with internally floating, and echogenic material in the breast parenchyma or retromammary region in tuberculosis breast. Magnetic resonance imaging may be useful in demonstrating the extramammary extent of disease. Other differential diagnosis that need to be kept in mind are actinomycosis of breast, which typically presents with discharge of sulphur granules from the sinus, however other fungal infections may not show such a picture and appropriate fungal staining and culture should be done for diagnosis.

Sarcoidosis of breast can present as a breast lump and biopsy may reveal non-caseating granulomas but sinus formation is rarely seen. Antitubercular therapy (ATT) with or without minimal surgical intervention forms the mainstay of treatment. Surgical intervention is required for diagnostic biopsy, aspiration of abscesses and excision of sinuses and masses.

CONCLUSION: Breast Tuberculosis although rare in clinical practice, is often underreported in literature and needs to kept as a differential diagnosis especially in high incidence areas. Also, the patients need to be counselled regarding complete treatment with ATT so that they do not stop medications and become a defaulter, which in turn increases the incidence of Multi Drug Resistance Tuberculosis. Biopsy and AFB smear are mainstay of diagnosis in Tuberculosis, however, AFB smear is often negative in Extrapulmonary Tuberculosis and interpretation of biopsy becomes difficult in partially treated cases.

\section{REFERENCES:}

1. Fanning A. Tuberculosis: 6. Extrapulmonary disease. CMAJ.1999; 160:1597-603.

2. Mukerjee $P$, George $M$, Maheshwari HB, Rao CP. Tuberculosis of the breast.

J Indian Med Assoc.1974; 62: 410-2.

3. Tewari M, Shukla HS. Breast tuberculosis: diagnosis, clinical features and management.

Ind J Med Res. 2005; 122:103-10.

4. Banerjee SN, Ananthakrishnan N, Mehta RB, Parkash S. Tuberculous mastitis: a continuing problem.World J Surg.1987;11(1):105-109.

5. Hale JA, Peters GN, Cheek JH. Tuberculosis of the breast: rare but still existent. Review of the literature and report of an additional case. Am J Surg.1985 Nov; 150(5); 620-24.

6. Bhatt GM and Austin HM. CT demonstration of empyema necessitates. J Comput Assist Tomogr. 1985;9:1108-9.
7. Yuen JHF, Lam TPW, Leong L. Primary tuberculosis of the breast. J HK Coll Radiol. 2003;6:33-35.

8. Kakkar S, Kapila K, Singh MK, Verma K. Tuberculosis of the breast: a cytomorphologic study. Acta Cytologica. 2000 May-Jun;44(3):292-6.

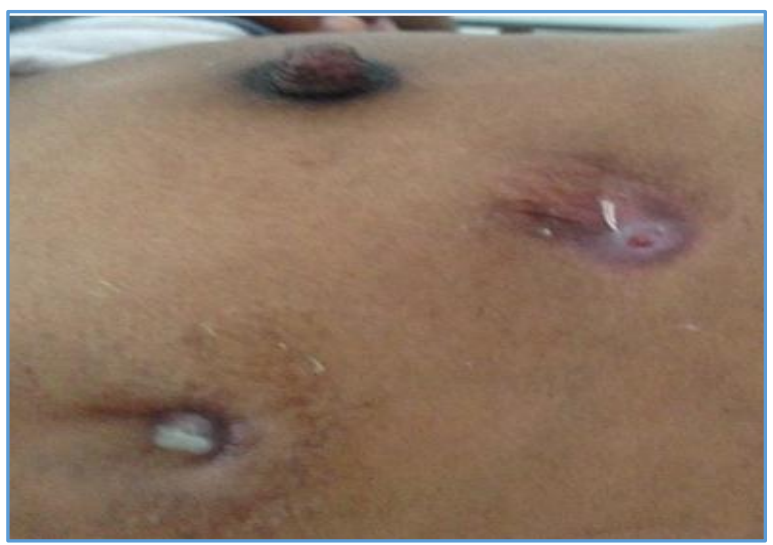

Fig. 1: Clinical Photograph of patient showing sinus opening on the right breast

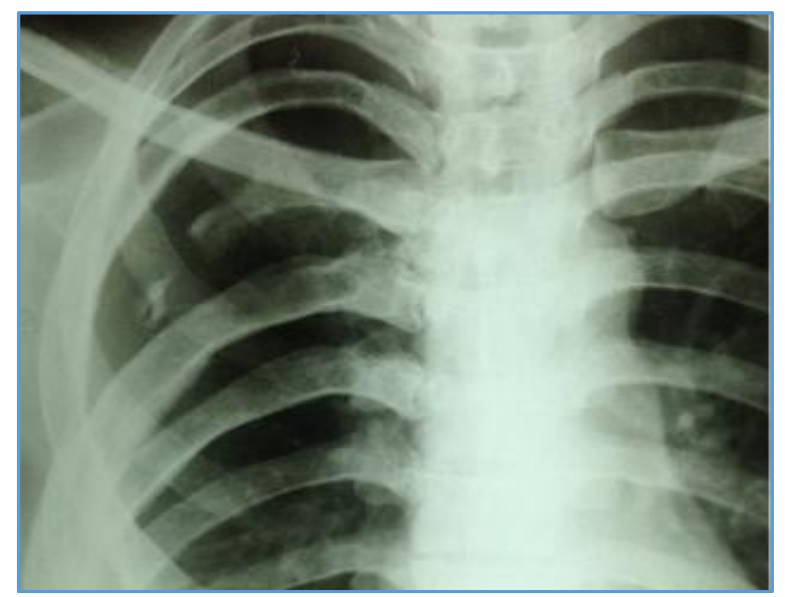

Fig. 2: Chest $X$-ray showing osteomyelitic changes in $5^{\text {th }}$ and $6^{\text {th }}$ ribs on right side with bony resorption of $5^{\text {th }}$ rib

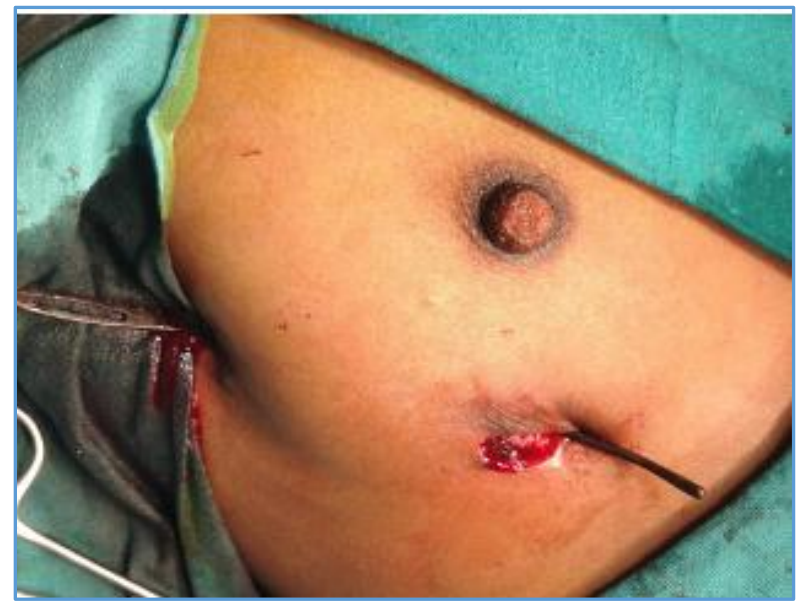

Fig. 3: Operative photograph demonstrating sinus tract in communication with ribs 